\title{
Corticosteroid therapy in IgA nephropathy with minimal proteinuria and high renal pathological score: A single-center cohort study
}

\author{
YUYAN TANG ${ }^{*}$, HAIDONG HE* ${ }^{*}$ WEIQIAN SUN, PIN HU, XIA CHEN and XUDONG XU \\ Department of Nephrology, Minhang Branch, Zhongshan Hospital, Fudan University, Shanghai 201199, P.R. China
}

Received January 21, 2018; Accepted July 12, 2018

DOI: $10.3892 / \mathrm{mmr} .2018 .9413$

\begin{abstract}
Currently, there is no clear evidence that advocates the widespread use of corticosteroids for the treatment of immunoglobulin Anephropathy( $\operatorname{IgAN}$ ) withminimalproteinuria $(<1 \mathrm{~g} /$ day $)$. The recent Kidney Disease: Improving Global Outcomes Clinical Practice Guideline recommends supportive corticosteroid treatment. In the present study, $45 \operatorname{IgAN}$ patients with high renal pathological scores and minimal proteinuria were enrolled. The patients were randomly divided into two groups. The treatment group received methylprednisolone tablets in addition to angiotensin-converting-enzyme inhibitor (ACE-I) and/or angiotensin-receptor blocker (ARB) treatment. The control group only received ACE-I and/or ARB treatment. In the treatment group, a single dose of $1 \mathrm{mg} / \mathrm{kg}$ (maximum $60 \mathrm{mg}$ /day) methylprednisolone tablets was given daily followed by gradually decreasing dosage. The follow-up time of the patients was 3 years. In addition, the underlying mechanisms were investigated. The results indicated that there was a significant reduction in the amount of urinary proteins in the treatment group compared with the control group. At the end of the follow-up, the endpoint event rate of moderate or severe proteinuria and decrease in estimated glomerular filtration rate (eGFR) in the treatment group was significantly lower than the control group. Furthermore, higher levels of serum cytokines, interleukin (IL)-4, IL-17, transforming growth factor- $\beta 1$ and IL-21, were detected in patients with IgAN compared with a group of healthy controls. There was no significant difference in IFN- $\gamma$ expression between the IgAN and healthy control groups. Furthermore, the expression of Janus kinase (Jak)1, Jak3, signal
\end{abstract}

Correspondence to: Dr Xudong Xu or Dr Yuyan Tang, Department of Nephrology, Minhang Branch, Zhongshan Hospital, Fudan University, 170 Xinsong Road, Shanghai 201199, P.R. China

E-mail:xxdmzx@sina.com

E-mail: tangyuyan361113248@163.com

${ }^{*}$ Contributed equally

Key words: immunoglobulin A nephropathy, renal pathological score, methylprednisolone tablets, Janus kinase/signal transducer and activator of transcription pathway, safety transducer and activator of transcription (STAT)3 and STAT6 was significantly upregulated in patients with IgAN compared with healthy controls. However, the expression levels of STAT5 and chaperone protein, C1GALT1 specific chaperone 1, in IgAN patients were significantly reduced compared with healthy controls. In addition, there was no significant difference in the expression of Jak2, tyrosine kinase 2, STAT1 and STAT4 between the two groups. In conclusion, for IgAN patients with minimal proteinuria and high renal pathological score corticosteroid therapy is likely to be effective. The dysregulation of serum cytokine levels in these patients with IgAN may have a role in the pathogenesis and progression of disease, which is associated with the activation of the JAK/STAT signaling pathway.

\section{Introduction}

Immunoglobulin A (IgA) nephropathy (IgAN) is the most common type of primary glomerulonephritis and a principal cause of end-stage renal disease (ESRD) worldwide. IgAN is a heterogeneous disease with different clinical and pathological phenotypes $(1,2)$; therefore, appropriate therapy for IgAN is debated among nephrologists. The recent Kidney Disease: Improving Global Outcomes (KDIGO) Clinical Practice Guidelines for IgA nephropathy recommend long-term angiotensin-converting enzyme (ACE) inhibitors or angiotensin-receptor blockers (ARBs) as treatment when proteinuria is $0.5-1 \mathrm{~g} / \mathrm{day}$ (3). However, after some time patients may experience relapse of proteinuria or more $(>1 \mathrm{~g} / \mathrm{day})$. These patients developed chronic renal insufficiency in the clinic, which indicated that treatment strategies that only depend on the severity of proteinuria were not comprehensive. Unfortunately, active treatment was frequently delayed until the late clinical stages of the disease, often beyond the time-point at which therapeutic intervention may be successful (4). In the current report, a single-center cohort study was designed to prospectively evaluate the efficiency and safety of corticosteroids for the treatment of IgA nephropathy patients with minimal proteinuria and high renal pathological score based on Haas classification ( $\geq$ type II), Katafuchi semi-quantitative integration method (score, $\geq 2$ ) and tubulointerstitial injury score ( $\geq 2$ points).

Renal biopsy is widely considered to be the gold standard for the diagnosis of IgAN (5). However, renal biopsy has potential 
complications that cannot be easily tolerated by the majority of patients, and repeated monitoring is technically difficult. Consequently, highly sensitive and specific non-invasive biomarkers that reflect disease severity and progression are urgently needed for the clinical management of patients with IgAN. A previous study demonstrated that galactose-deficient IgA1 (Gd-IgA1) is one of the key effector molecules in the pathogenesis of IgAN (6). Unfortunately, the underlying molecular mechanisms are still under extensive investigation.

The synthesis of O-glycans starts from the addition of $\mathrm{N}$-acetylgalactosamine to a peptide catalyzed by the enzyme $\mathrm{N}$-acetylgalactosaminyltransferase 2 (CSGALNACT2), and continues with the addition of galactose by core $1 \mathrm{bl}$-3-galactosyltransferase (C1GALT1). C1GALT1 specific chaperone 1 (Cosmc) is essential for the activity of the mammalian C1GALT(7). The level of Cosmcin B-lymphocytes was reported to be lower in patients with IgAN than in normal controls, and the level of Cosmc was negatively correlated with the level of aberrant glycosylation of IgA1 (8). Galactose-deficient IgA1 (Gd-IgA1) was reported to be elevated in metabolites that were excreted in the urine of patients with $\operatorname{IgAN}$ and the levels of urinary Gd-IgA1 were correlated with proteinuria (9). However, as sample preparation (isolation of the IgA1 hinge region) is complicated, the current techniques for detecting Gd-IgA1 are not suitable for clinical application. Furthermore, an increased frequency of B-lymphocytes was observed, particularly in patients with an elevated serum concentration of Ig A (10). Notably, polymeric IgA1 is secreted by active polyclonal B-lymphocytes, and T-lymphocytes are involved in the secretion of IgA by B-lymphocytes.

Lymphocytes primed by antigens at mucosal sites produce abnormal amounts of deglycosylated IgA1 and polymeric IgA-IgG immunocomplexes $(11,12)$. T-lymphocytes are critical in the control of the antigen-driven adaptive immune response. In particular, the polarization of T-helper cells can affect IgA nephropathy $(13,14)$. In addition, each of these T-cell subtypes is characterized by certain specialized cytokines and has various immune functions that are dependent on the type of cytokine (15). Therefore, the dysregulation of T-cells may cause B-cells to secrete Gd-IgA1, which deposits in the mesangium and triggers several immune and pathological changes, culminating in the development of $\operatorname{Ig} \mathrm{A}$ nephropathy. In the current study, the levels of T-cell cytokines, Janus kinase (JAK)/signal transducer and activator of transcription (STAT) pathway proteins and the chaperone protein, COSMC, were analyzed in peripheral blood mononuclear cells (PBMCs) of patients with IgAN prior to treatment and in the normal control group. A group of 49 healthy subjects, recruited at the Minhang Branch of Zhongshan Hospital (Shanghai, China), were selected as the normal control group. Changes in these indicators changed were analyzed, and whether these indicators could predict the severity of kidney disease in IgA nephropathy patients with minimal proteinuria and high renal pathological scores was determined.

\section{Materials and methods}

Consent and ethics approval. All healthy and patients donors provided written informed consent prior to sampling. The experiments and procedures were conducted in accordance with the Helsinki Declaration of 1975, and were approved by the Human Ethics Committee of School of Medicine, Fudan University (Shanghai, China).

Inclusion criteria of patients. A total of 45 patients with $\operatorname{Ig} \mathrm{A}$ nephropathy were included in the study. The inclusion criteria were as follows: Diagnosis of IgA nephropathy as confirmed by renal biopsy with minimal proteinuria (0.5-1.0 g/day); with (or without) microscopic hematuria and estimated glomerular filtration rate $(\mathrm{eGFR}) \geq 90 \mathrm{ml} / \mathrm{min} / 1.73 \mathrm{~m}^{2}$; and biopsy findings that include Haas grading of pathological classification $\geq$ type II (16), glomerular injury score of Katafuchi integral $\geq 2$ and/or tubulointerstitial injury score $\geq 2$ points (17). The treatment group ( 22 cases) was administered with hormone and conventional treatment (ACE-I and/or ARB). The control group (23 cases) only received conventional therapy (ACE-I and/or ARB). The clinical characteristics of the patients are listed in Table I.

Treatment protocol. Patients underwent renal biopsy prior to treatment. The patients were randomly divided into two groups. The treatment group (22 cases) received methylprednisolone tablets and ACE-I (Lotensin $10 \mathrm{mg} / \mathrm{day}$ ) and/or ARB treatment (Losartan $50 \mathrm{mg} /$ day) for 3 years. A single daily dose of $1 \mathrm{mg} / \mathrm{kg}$ (maximum $60 \mathrm{mg} /$ day) methylprednisolone tablets was given to the treatment group, which was gradually decreased. When the dose of methylprednisolone was reduced to $10 \mathrm{mg} / \mathrm{day}$, the dose was maintained for 6 months and then further reduced to $5 \mathrm{mg} /$ day for another 6 months. The control group (23 cases) only received ACE-I and/or ARB treatment. The levels of T-cell cytokines and molecules involved in the JAK/STAT pathway and the chaperone protein, COSMC, were detected in the PBMCs of patients with IgAN prior to treatment and in the normal control group. For both groups, if the blood pressure increased, a calcium channel blocker, Norvasc ( $5 \mathrm{mg} / \mathrm{day}$ ), was used to control blood pressure $(130 / 80 \mathrm{mmHg})$. The duration of patient follow-up was 3 years.

Laboratory data. The blood pressure of all patients was measured daily prior to and following treatment. Other tests, including 24-h urinary protein, quantitative analysis of urine sediment, renal function, blood lipid, blood glucose, hemoglobin subunit $\alpha 1$ and eGFR, were also performed monthly prior to and following treatment. eGFR was evaluated using the CKD-EPI equation (18): eGFR $\left(\mathrm{ml} / \mathrm{min} / 1.73 \mathrm{~m}^{2}\right)=141 \mathrm{x} \min$ [serum creatinine $(\mathrm{SCr} / \mathrm{k}, 1)] \alpha \times \max (\mathrm{SCr} / \mathrm{k}, 1)-1.209 \times 0.993$ age $x 1.018$ (if female) x 1.159 (if of African descent). The value of $\mathrm{k}$ is 0.7 for females and 0.9 for males. The value of $\alpha$ is -0.329 for females and -0.411 for males. Min refers to the minimum $\mathrm{SCr} / \mathrm{k}$ and 1 , and max refers to the maximum $\mathrm{SCr} / \mathrm{k}$ and 1 . All blood samples from patients with $\mathrm{Ig} \mathrm{AN}$ were collected prior to renal biopsy and treatment. The participants in the control group were from patients that underwent physical examinations at the hospital.

Cell isolation. PBMCs were collected from fresh heparinized blood by the use of the Ficolle Isopaque gradient centrifugation prior to renal biopsy. Briefly, $5 \mathrm{ml}$ peripheral venous blood samples were obtained from patients and healthy subjects in 
Table I. Clinical characteristics of patients.

\begin{tabular}{|c|c|c|c|c|c|}
\hline Characteristic & $\begin{array}{c}\text { Treatment group } \\
\text { (methylprednisolone and } \\
\text { ACE-I and/or ARB treatment) }\end{array}$ & $\begin{array}{l}\text { Control group } \\
\text { (ACE-I and/or } \\
\text { ARB treatment) }\end{array}$ & P-value ${ }^{a}$ & $\begin{array}{l}\text { Healthy } \\
\text { subjects }\end{array}$ & P-value ${ }^{a}$ \\
\hline Number & 22 & 23 & & 49 & \\
\hline Sex (male/female) & $12 / 10$ & $11 / 12$ & & $26 / 23$ & \\
\hline Age (years) & $35.12 \pm 6.10$ & $34.50 \pm 7.10$ & 0.736 & $39.32 \pm 7.20$ & 0.816 \\
\hline Smoking (n) & 4 & 3 & & 6 & \\
\hline Drinking (n) & 2 & 2 & & 4 & \\
\hline Course of disease (months) & $4.51 \pm 1.03$ & $4.09 \pm 1.28$ & 0.096 & & \\
\hline Systolic pressure (mmHg) & $126.14 \pm 21.27$ & $130.08 \pm 24.70$ & 0.471 & $125 \pm 5.27$ & 0.541 \\
\hline Diastolic pressure (mmHg) & $74.82 \pm 14.25$ & $77.24 \pm 13.91$ & 0.474 & $70 \pm 10.25$ & 0.128 \\
\hline HBA1c $(\%)$ & $5.33 \pm 0.60$ & $5.84 \pm 0.52$ & 0.999 & $5.23 \pm 3.15$ & 0.130 \\
\hline $\mathrm{FBG}(\mathrm{mmol} / \mathrm{l})$ & $5.12 \pm 1.44$ & $4.86 \pm 1.32$ & 0.410 & $4.58 \pm 2.34$ & 0.147 \\
\hline $\mathrm{TG}(\mathrm{mmol} / \mathrm{l})$ & $4.61 \pm 0.31$ & $5.07 \pm 0.40$ & 0.909 & $4.68 \pm 0.65$ & 0.087 \\
\hline $\mathrm{TC}(\mathrm{mmol} / \mathrm{l})$ & $2.02 \pm 0.24$ & $2.06 \pm 0.17$ & 0.525 & $2.14 \pm 0.68$ & 0.098 \\
\hline $\mathrm{eGFR}\left(\mathrm{ml} / \mathrm{min} / 1.73 \mathrm{~m}^{2}\right)$ & $94.15 \pm 11.57$ & $93.13 \pm 10.51$ & 0.824 & $112 \pm 10.56$ & 0.076 \\
\hline 24 h Upro (g/day) & $0.88 \pm 0.15$ & $0.82 \pm 0.11$ & 0.094 & $0.2 \pm 0.58$ & 0.032 \\
\hline Microscopic hematuria (/HP) & $22.31 \pm 10.20$ & $25.13 \pm 13.11$ & 0.626 & & \\
\hline
\end{tabular}

${ }^{\mathrm{a}}$ Vs. treatment group. ACE-I, angiotensin-converting-enzyme inhibitor; ARB, angiotensin-receptor blocker; FBG, fasting blood glucose; HBA1c, glycated hemoglobin; HP, high-power field; TG, triglyceride; TC, total cholesterol; eGFR, estimated glomerular filtration rate; Upro, urinary protein.

a sterile heparinized test tube. The whole blood sample was diluted to a final volume of $10 \mathrm{ml}$ by phosphate-buffered saline (PBS) in a 15-ml centrifuge tube. The diluted blood was then slowly poured into Ficoll-Biocoll separating solution $(5 \mathrm{ml}$; Dakewe Biotech Co., Ltd., Beijing, China) in a centrifuge tube. Finally, PBMCs were isolated following density gradient centrifugation $\left(1,007.1 \mathrm{x} \mathrm{g}, 20^{\circ} \mathrm{C}, 20 \mathrm{~min}\right)$. The isolated PBMCs were then washed twice with sterile PBS (566.5 x g, $20^{\circ} \mathrm{C}, 10 \mathrm{~min}$ ) and carefully re-suspended in RPMI-1640 medium (Gibco; Thermo Fisher Scientific, Inc., Waltham, MA, USA) in 6-well plate. Cell viability was estimated by trypan blue staining, and cell viability should always be $>95 \%$. The cells were counted, and the final cell density was adjusted to $5-6 \times 10^{6}$ cells $/ \mathrm{ml}$. The PBMCs from healthy donors were collected in the same manner. Meanwhile, serum samples were isolated from $5 \mathrm{ml}$ blood prior to renal biopsy, which was centrifuged for $20 \mathrm{~min}$ at 2,389.5 x g to obtain $2 \mathrm{ml}$ plasma. The plasma samples were added to a 24 -well culture plate and maintained at $-20^{\circ} \mathrm{C}$ for subsequent experiments. The serum samples from the normal control group were collected in the same manner.

Analysis of the levels of cytokines. The concentrations of serum interferon- $\gamma$ (IFN- $\gamma$; cat. no. EH0195), interleukin (IL)-4 (cat. no. EH0212), IL-17 (cat. no. EH0228), IL-21 (cat. no. EH0435) and TGF- $\beta 1$ (cat. no. EH0304) were measured using ELISA kits (Shanghai Weiao Biotechnology Co., Ltd., Shanghai, China; http:/www.biotechwell.com), following the manufacturer's protocols. The levels of cytokines were determined by measuring absorbance at $450 \mathrm{~nm}$ with a microplate reader. The concentrations of the cytokines in the samples were calculated using standard curves. The data are expressed as $\mathrm{pg} / \mathrm{ml}$.

Analysis of COSMC and JAK/STAT pathway genes by reverse transcription-quantitative polymerase chain reaction $(R T-q P C R)$. Total RNA was extracted from the harvested PBMCs according to the protocol of the TRIzol reagent (Invitrogen; Thermo Fisher Scientific, Inc.). cDNA was synthesized by RT using the PrimeScript 1st Strand cDNA Synthesis kit (Takara Bio, Inc., Otsu, Japan). qPCR reactions were prepared using SYBR Premix Ex Taq ${ }^{\mathrm{TM}}$ reagents (Takara Bio, Inc.) on a LightCycler (Roche Diagnostics, Basel, Switzerland). The PCR cycling conditions were as follows: $30 \mathrm{sec}$ at $95^{\circ} \mathrm{C}$ followed by 40 cycles of $95^{\circ} \mathrm{C}$ for $5 \mathrm{sec}$ and $60^{\circ} \mathrm{C}$ for $30 \mathrm{sec}$, and a final step of $95^{\circ} \mathrm{C}$ for $15 \mathrm{sec}, 60^{\circ} \mathrm{C}$ for $1 \mathrm{~min}$ and $95^{\circ} \mathrm{C}$ for $15 \mathrm{sec}$. GAPDH served as the internal reference. The sequences of the PCR primers used are as follows: JAK1 forward, 5'-TGCTCC TGAGTGTGTTGAGG-3' and reverse, 5'-AGGTCAGCC AGCTCCTTACA-3'; JAK2 forward, 5'-GAGCCTATC GGCATGGAATA-3' and reverse, 5'-ACTGCCATCCCA AGACATTC-3'; JAK3 forward, 5'-TCTCAAGGAGCA GGGTGAGT-3' and reverse, 5'-GTAGGCAGGCCTTGT AGCTG-3'; tyrosine kinase 2 (Tyk2) forward, 5'-TGACCC TGTATGAGCTGCTG-3' and reverse, 5'-CTGTCATCT GACCCTGAGCA-3'; STAT1 forward, 5'-TTCAGGAAG ACCCAATCCAG-3' and reverse, 5'-TGAATATTCCCC GACTGAGC-3'; STAT4 forward, 5'-AGCCTTGCGAAG TTTCAAGA-3' and reverse, 5'-ACACCGCATACACAC TTGGA-3'; STAT5 forward, 5'-ACATTTGAGGAGCTG CGACT-3' and reverse, 5'-CCTCCAGAGACACCTGCT 
Table II. Pathological characteristics of patients.

\begin{tabular}{lccr}
\hline Characteristic & Treatment group & Control group & P-value \\
\hline Haas II type (n) & 11 & 12 & 0.814 \\
Haas III type (n) & 8 & 9 & 0.956 \\
Haas IV type (n) & 3 & 2 & 0.992 \\
Glomerular score & & & \\
Glomerular cell proliferation score & $1.22 \pm 0.61$ & $1.14 \pm 0.52$ & 0.501 \\
Segmental lesion score & $0.83 \pm 0.30$ & $0.91 \pm 0.44$ & 0.705 \\
Glomerulosclerosis score & $0.72 \pm 0.41$ & $0.64 \pm 0.32$ & 0.295 \\
Renal tubule interstitial score & & & \\
Percentage of lesions & $1.03 \pm 0.51$ & $1.12 \pm 0.61$ & 0.499 \\
Inflammatory cell infiltration & $0.74 \pm 0.42$ & $0.63 \pm 0.32$ & 0.295 \\
Interstitial fibrosis & $0.61 \pm 0.20$ & $0.60 \pm 0.31$ & 0.090 \\
Renal tubular atrophy & $0.62 \pm 0.30$ & $0.71 \pm 0.40$ & 0.705 \\
\hline
\end{tabular}

Table III. Clinical characteristics of patients following treatment.

\begin{tabular}{lccr}
\hline Characteristic & Treatment group & Control group & P-value \\
\hline Systolic pressure $(\mathrm{mmHg})$ & $128.14 \pm 15$ & $130.44 \pm 17.34$ & 0.336 \\
Diastolic pressure $(\mathrm{mmHg})$ & $79.90 \pm 15.32$ & $80.43 \pm 16.74$ & 0.078 \\
HBAlc $(\%)$ & $5.90 \pm 1.13$ & $5.34 \pm 0.92$ & 0.067 \\
FBG $(\mathrm{mmol} / \mathrm{l})$ & $5.84 \pm 1.62$ & $5.33 \pm 1.1$ & 0.257 \\
TG $(\mathrm{mmol} / \mathrm{l})$ & $5.22 \pm 0.43$ & $5.10 \pm 0.56$ & 0.452 \\
TC $(\mathrm{mmol} / \mathrm{l})$ & $2.45 \pm 0.31$ & $2.28 \pm 0.22$ & 0.052 \\
eGFR $\left(\mathrm{ml} / \mathrm{min} / 1.73 \mathrm{~m}^{2}\right)$ & $85.62 \pm 12.22$ & $80.56 \pm 13.39$ & 0.410 \\
24 h Upro $(\mathrm{g} /$ day) & $0.48 \pm 0.17$ & $0.93 \pm 0.36$ & 0.001 \\
Microscopic hematuria (/HP) & $19.42 \pm 15.30$ & $33.41 \pm 16.70$ & 0.991
\end{tabular}

FBG, fasting blood glucose; HBA1c, glycated hemoglobin; HP, high-power field; TG, triglyceride; TC, total cholesterol; eGFR, estimated glomerular filtration rate; Upro, urinary protein.

TC-3'; STAT6 forward, 5'-CAACCACTTCCTACCCCA GA-3' and reverse, 5'-ATGCTCATGGAGGAATCAGG-3'; COSMC forward, 5'-TTTGAAGGGTGTGATGCTTG-3' and reverse, 5'-ATGCGCTCATCCTCTGAAAT-3'; GAPDH forward, 5'-GCGAGATCCCTCCAAAATCAA-3' and reverse, 5'-GTTCACACCCATGACGAACAT-3'.

Safety endpoints. Patients were withdrawn from the study if they experienced moderate proteinuria or more (proteinuria $>1 \mathrm{~g} /$ day) and decline in eGFR (eGFR $<90 \mathrm{ml} / \mathrm{min} / 1.73 \mathrm{~m}^{2}$ ). The duration of follow-up for patients was 3 years. During the entire study, no patient reached the safety endpoints.

Statistical analyses. The data are presented as the mean \pm standard error and were analyzed using the SPSS 19.0 software (IBM Corp., Armonk, NY, USA). One-way analysis of variance and Student-Newman-Keuls test were used to analyze the data. The t-test was used to determine the statistical significance in comparison to two groups. Kaplan-Meier was used for prognostic survival analysis and the log-rank test was used to compare survival in the two groups. The Haas classification data were analyzed using $\chi^{2}$ test. $\mathrm{P}<0.05$ was considered to indicate a statistically significant difference.

\section{Results}

Clinical characteristics of the subjects. There were no statistically significant differences between the two groups (treatment and control group) in sex, age, duration of disease, long-term smoking and drinking, pathological classification and integrity of kidney, blood glucose, blood pressure, blood lipid, $24 \mathrm{~h}$ urine protein quantification, microscopic hematuria and eGFR. The clinical characteristics of the patients are listed in Table I, and the pathological characteristics are listed in Table II.

Efficiency. There was a significant reduction in the level of urinary protein in the methylprednisolone treatment group compared with the control treatment group $(\mathrm{P}<0.05)$, whereas there was no significant difference in blood glucose, blood lipid and blood pressure (Table III).

Survival analysis. The renal outcome endpoint was selected as reaching a moderate amount of proteinuria or more 


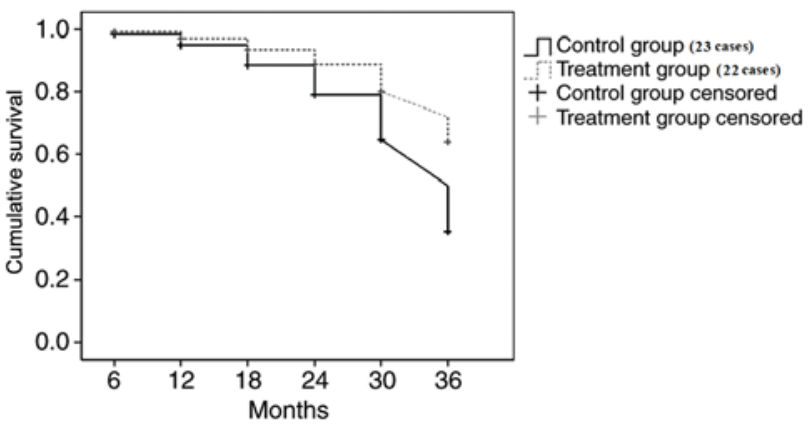

Figure 1. Prognosis of proteinuria survival curves. The renal outcome endpoint was moderate proteinuria or more ( $>1 \mathrm{~g} /$ day) and estimated glomerular filtration rate $<90 \mathrm{ml} / \mathrm{min} / 1.73 \mathrm{~m}^{2}$. Patient follow-up time was 3 years. The endpoint event rate of moderate proteinuria or more in the treatment group (received methylprednisolone tablets and ACE-I and/or ARB treatment) was significantly lower than that in the control group (only received ACE-I and/or ARB treatment; $\mathrm{P}<0.05$ by log-rank test). ACE-I, angiotensin-converting-enzyme inhibitor; $\mathrm{ARB}$, angiotensin receptor blocker.

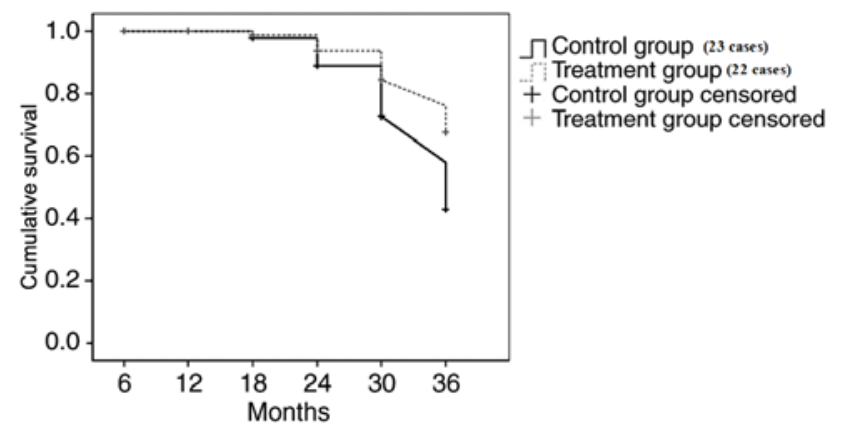

Figure 2. Prognosis of eGFR survival curves. The renal outcome endpoint was moderate proteinuria or more ( $>1 \mathrm{~g} /$ day) and eGFR decline $<90 \mathrm{ml} / \mathrm{min} / 1.73 \mathrm{~m}^{2}$. Patients follow-up time was 3 years. The endpoint event rate of eGFR decline in the treatment group (received methylprednisolone tablets and ACE-I and/or ARB treatment) was significantly lower than that in the control group (only received ACE-I and/or ARB treatment; $\mathrm{P}<0.05$ by log-rank test). eGFR, estimated glomerular filtration rate; ACE-I, angiotensin-converting-enzyme inhibitor; $\mathrm{ARB}$, angiotensin receptor blocker.

$\left(>1 \mathrm{~g} /\right.$ day) and eGFR (eGFR $\left.<90 \mathrm{ml} / \mathrm{min} / 1.73 \mathrm{~m}^{2}\right)$. The follow-up time of patients was 3 years. At the end of the follow-up, the endpoint event rate of moderate amount of proteinuria or more and eGFR decline in the methylprednisolone treatment group was significantly lower than the control treatment group $(\mathrm{P}<0.05$; Figs. 1 and 2$)$.

Levels of serum cytokines, $I F N-\gamma, I L-4, I L-17$, and $I L-2 I$ and TGF- $\beta 1$. The current consensus is that IgAN is the most common primary glomerulonephritis and is caused by immune factors. Emerging evidence suggests that the imbalance of $\mathrm{T}$ cells pro-inflammatory cytokines has a crucial role in the development and progression of $\operatorname{IgAN}(12,13)$. Therefore, the serum levels of cytokines, IL-4, IL-17, IL-21 and TGF- $\beta 1$, from patients with $\operatorname{IgA}$ nephropathy were assayed. Higher levels of serum $(31.51 \pm 15.56)$, IL-17 $(30.69 \pm 12.85)$, TGF- $\beta 1$ $(203.06 \pm 66.63)$ and IL-21 $(89.03 \pm 34.83)$ were detected in IgAN patients compared with healthy subjects. However, there was no statistical difference in the levels of IFN- $\gamma$ between IgAN patients $(6.96 \pm 1.44 \mathrm{pg} / \mathrm{ml})$ and the healthy control group $(7.31 \pm 3.28 \mathrm{pg} / \mathrm{ml} ; \mathrm{t}=0.26, \mathrm{P}=0.796)$. After 1 month, the levels of serum IL-4 (11.11 \pm 1.41$)$, IL-17 (14.94 \pm 2.57$)$, TGF- $\beta 1$ $(70.11 \pm 13.01)$ and IL-21 $(62.96 \pm 8.70)$ in the treatment group were decreased compared with the levels prior to treatment $(\mathrm{P}<0.01$; Fig. 3).

Levels of the chaperone protein Cosmc. Research has demonstrated that the expression level of Cosmc in B-lymphocytes was lower in patients with $\operatorname{IgAN}$ compared with normal controls, and the level of Cosmc was negatively correlated with the expression of galactose-deficient IgA1 (8). The mRNA expression of COSMC, a chaperone protein, was analyzed from the PBMCs of IgAN patients. The mRNA expression of Cosmc was decreased in IgAN patients compared with healthy controls $(\mathrm{P}<0.01)$. The mRNA levels of Cosmc in patients that were treated for 1 month were increased compared with the levels prior to treatment ( $\mathrm{P}=0.0233$; Fig. 4).

Expression of proteins that are associated with the JAK/STAT pathway. The JAK/STAT pathway is the principal signaling mechanism for a wide array of cytokines and growth factors. To analyze the mechanism of galactose-deficient IgA1 in IgAN, the expression of Jak1, Jak2, Jak3, Tyk2, STAT1, STAT3, STAT4, STAT5 and STAT6 mRNA was analyzed in the PBMCs of patients with IgAN before treatment and healthy controls. The results revealed that the expression of Jak1, Jak3, STAT3 and STAT6 mRNA was significantly upregulated in the PBMCs of IgAN patients. However, the level of STAT5 mRNA was decreased in the PBMCs of patients IgAN compared with healthy controls. However, there was no significant difference in the levels of Jak2, Tyk2, STAT1 and STAT4 mRNA expression between the IgAN patients and the healthy control group (P>0.05; Fig. 5).

\section{Discussion}

IgAN is an immune complex-mediated disease, as circulating immune complexes are deposited exclusively in the glomerular mesangium, which leads to the occurrence and development of IgAN. The circulating immune complexes are mainly composed of Gd-IgA1 and IgG anti-Gd-IgA1 antibodies (19). Microscopic hematuria and proteinuria are the most common presentations of IgAN (20).

Renal biopsy is considered to be the gold standard for the diagnosis of IgAN. Emerging evidence suggests that the presence of histopathologic lesions to be risk factors for the development and progression of IgA nephropathy. In the past few decades, various histological parameters have been used to predict the prognosis of patients with IgAN (19). To date, pathological classifications of IgAN included glomerular score, Lee's classification and Haas classification (21). Each of the classifications has limitations. The semi-quantitative glomerular score system encompasses three pathologic lesions associated with progression, including glomerular hypercellularity (mesangial and endocapillary), segmental lesions (such as tuft adhesion, segmental sclerosis and crescent) and global glomerular sclerosis (17). In the present study, the indices of each lesion were semi-quantitatively determined. The glomerular score is also known as the Katafuchi semi-quantitative integral, and it is generally 
A
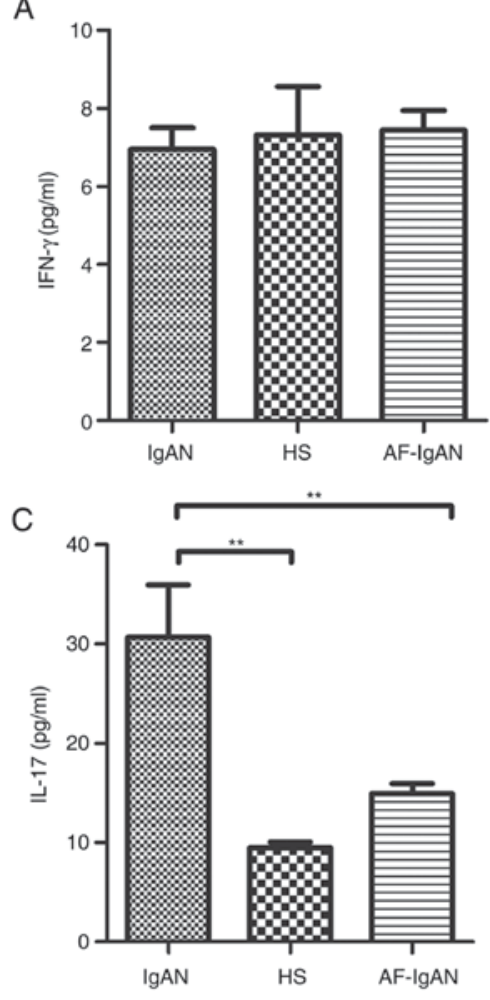

B

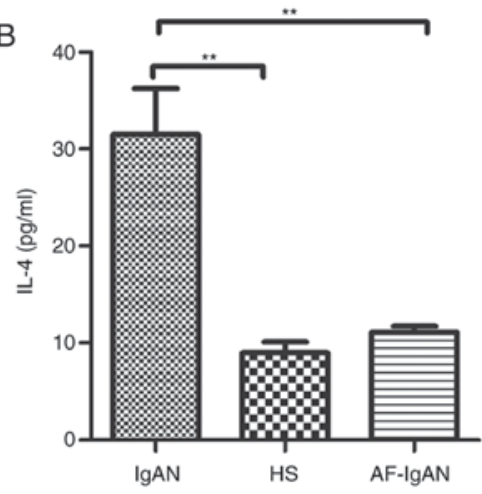

D

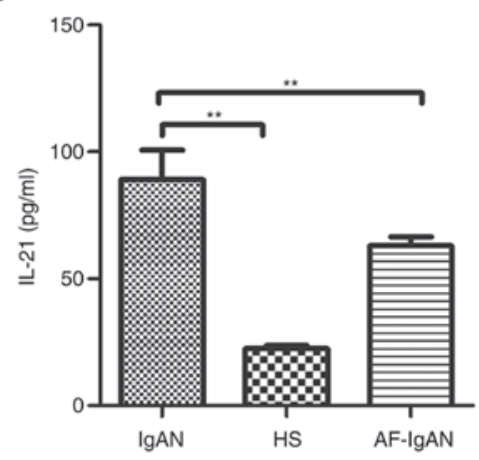

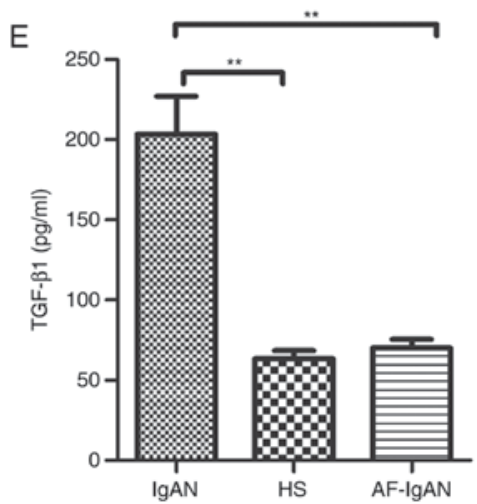

Figure 3. Analysis of serum cytokines in patients with IgAN. The levels of serum (A) IFN- $\gamma$, (B) IL-4, (C) IL-17, (D) IL-21 and (E) TGF- $\beta 1$ in subjects were analyzed by ELISA. Data are expressed as the mean \pm standard deviation of individual samples from three separate experiments. The concentrations of serum IL-4, IL-17, TGF- $\beta 1$ and IL-21 in the IgAN patients were significantly higher than that in the HS ( $<<0.01)$. There was no significant difference on the expressions of the IFN- $\gamma$ between in IgAN patients and the healthy control group $(\mathrm{t}=0.26, \mathrm{P}=0.796)$. After 1 month of treatment with methylprednisolone and ACE-I and/or ARB treatment, the levels of serum IL-4, IL-17, TGF- $\beta 1$ and IL-21 were reduced compared with prior to treatment. ${ }^{* *} \mathrm{P}<0.01$. IFN- $\gamma$, interferon- $\gamma$; IgAN, immunoglobulin A nephropathy; HS, healthy subjects; AF-IgAN, IgAN patients 1 month post-treatment with methylprednisolone and ACE-I and/or ARB; IL, interleukin; TGF- $\beta 1$, transforming growth factor- $\beta 1$.

closely associated with the renal outcome (17). The Lee's and Haas classifications are also known as single-grade scoring systems, which have been widely used in the clinical practice $(22,23)$. In the present study, all three histologic classifications, Haas classification, Katafuchi semi-quantitative integral glomerular injury score and tubulointerstitial injury score, were used. In this study, a high renal pathological score considered as Haas classification $\geq$ type II, Katafuchi semi-quantitative integral $\geq 2$ and/or tubulointerstitial injury score $\geq 2$ points, and this was used as a criterion for recruitment of patients.

KDIGO used of $<1 \mathrm{~g} /$ day urinary protein as a standard for determining the prognosis of patients with primary glomerulonephritis (24). However, KDIGO did not provide a recommended treatment for IgAN patients with asymptomatic hematuria and urine protein in the range of 0.15-1 g/day. KDIGO suggests non-specific supportive treatment (particularly renin-angiotensin system blocking agents) for these patients (grade A, level $1 \mathrm{~b}$ ). However, there is currently no clear evidence that advocates the widespread use of corticosteroids for the treatment of IgAN with minimal proteinuria $(<1 \mathrm{~g} /$ day) (3). Studies have suggested that the incidence of renal interstitial vascular disease in IgAN was high, and mild clinical manifestations of IgAN in parents may predispose offspring to severe renal pathological lesions, which may lead to severe renal dysfunction (25-27). Therefore, guiding the treatment of IgAN according to clinical proteinuria has deficiencies. 


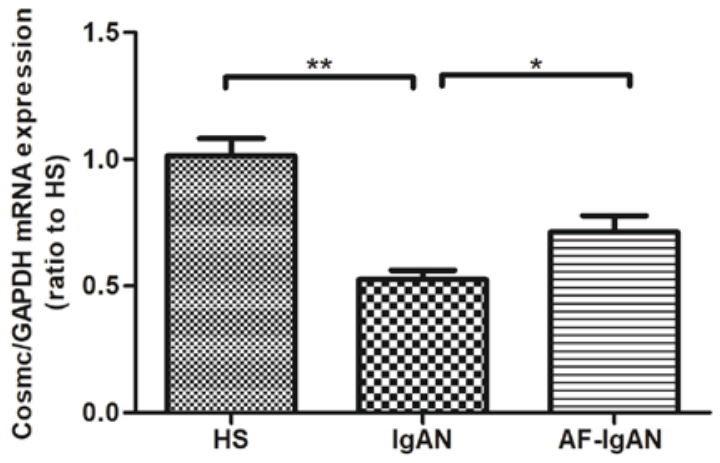

Figure 4. mRNA expression of the chaperone protein Cosmc in patients with IgAN. Chaperone protein Cosmc mRNA was decreased in patients IgAN compared with HS $(\mathrm{P}<0.01)$, The IgAN patients were treated with methylprednisolone and ACE-I and/or ARB for 1 month, then the levels of Cosmc mRNA was increased compare with prior to treatment $(\mathrm{P}=0.0233)$. Data are presented as the mean \pm standard deviation $\left({ }^{*} \mathrm{P}<0.05 ;{ }^{* *} \mathrm{P}<0.01\right)$. Cosmc C1GALT1 specific chaperone 1; IgAN, immunoglobulin A nephropathy; HS, healthy subjects; AF-IgAN, IgAN patients 1 month post-treatment with methylprednisolone and ACE-I and/or ARB

In the absence of optimal and comprehensive data from randomized trials of IgAN, the Supportive vs. Immunosuppressive Therapy of Progressive IgA Nephropathy (STOP-IgAN) trial has been conducted. The STOP-IgAN trial aimed to investigate whether immunosuppressive agents are effective for patients with high-risk IgAN. In the STOP-IgAN study, systemic steroid/immunosuppressive treatment significantly decreased proteinuria, but did not stop disease progression $(28,29)$. In the present study, patients with minimal proteinuria $(<1 \mathrm{~g} /$ day) and a high renal pathological score, that underwent conventional therapy (ACE-I and/or ARB treatment) and methylprednisolone corticosteroid therapy, were selected for recruitment. The endpoint for renal outcome was a moderate level of proteinuria and a high renal pathological score, who were given conventional therapy (ACE-I and/or ARB treatment) and corticosteroid therapy. The moderate amount of proteinuria or more was $>1 \mathrm{~g}$ /day and eGFR decline $<90 \mathrm{ml} / \mathrm{min} / 1.73 \mathrm{~m}^{2}$. The follow-up time of patients was 3 years. At the end of the follow-up, the endpoint event rate of moderate proteinuria or more, and eGFR decline in the methylprednisolone treatment group was significantly lower than the control group that did not receive methylprednisolone $(\mathrm{P}<0.05)$. In addition, the results demonstrated the importance of obtaining biopsies in the clinical management of IgAN, as the pathological changes in the kidneys were serious in some patients with minimal proteinuria ( $<1 \mathrm{~g}$ /day). In $\operatorname{IgAN}$ patients with small amount of proteinuria $(<1 \mathrm{~g} /$ day), for decisions on treatment options-whether to select conservative treatment involving ACE inhibitors or ARBs and whether to use combined therapy-clinicians should base the decision for treatment on the pathological grading, rather than proteinuria alone.

Renal biopsy has potential complications and repeated monitoring is technically difficult. Therefore, a clearer understanding of the molecular mechanisms should facilitate the identification of specific non-invasive biomarkers that reflect disease severity and progression. $\operatorname{IgAN}$, one of the most common types of primary glomerulopathy globally, is characterized by the glomerular mesangial deposition of Gd-IgA1 in the kidney (6). The IgA1 hinge region is composed of the hypogalactosylated $\mathrm{O}$-glycosides that result in an increased tendency for non-covalent self-aggregation and polymerization of circulating IgA1. Several studies have supported that Gd-IgA1 has a pivotal role in renal tissue injury (30). The origin of this galactosylation defect remains unclear. Increased synthesis of Gd-IgA1 may be the result of an imbalance between the activities of enzymes involved in post-translational galactosylation in the Golgi apparatus $(31,32)$.

The synthesis of O-glycans begins with the addition of $\mathrm{N}$-acetylgalactosamine to a peptide catalyzed by the enzyme CSGALNACT2, which then continues with the addition of galactose by the enzyme C1GALT1 (33). Furthermore, C1GALT1 has been shown to be assisted by the chaperone protein, Cosmc, which is crucial for ensuring the stability and enzymatic activity of galactosyltransferase $(34,35)$. Notably, Cosmc expression is decreased in the B-cells of patients with IgAN and is negatively correlated with Gd-IgA1 (8). In the current study, the level of Cosmc mRNA was analyzed in the PBMCs from patients with IgAN by RT-qPCR. The mRNA expression of Cosmc was lower in IgAN patients compared with healthy control subjects.

The synthesis of Gd-IgA1 is associated with imbalanced activity between enzymes, including C1GALT1 and the dysregulation of $\mathrm{CD}^{+}{ }^{+} \mathrm{T}$-cell subset. Accumulating evidence suggests that $\mathrm{Gd}-\mathrm{Ig} \mathrm{A} 1$ deposits may be produced from mucosal plasma cells, and associated with T-cell dysregulation (36). There is a growing body of evidence indicating that an impaired mucosal IgA response may lead to the impaired depletion of mucosal antigens (36). Mucosal immunity depends on the equilibrium between the responsiveness and tolerance of antigens, and the $\mathrm{CD}^{+} \mathrm{T}$-cell subset has a key role in maintaining or disrupting this delicate balance (37). Previous studies have demonstrated that the predominant cytokines secreted by T-lymphocytes in IgAN were the Th2 type, and that the Th2 cytokine, IL-4, may have a critical role in leading the glycosylation of the IgA1 hinge region (38) and renal fibrosis (39); this cytokine production may lead to the overproduction of Gd-IgA1, which is prone to deposition in the mesangium (40). Strong polarization toward the production of Th1 type cytokines in IgAN was also indicated by another study (41), and Th1 predominance was reported to be associated with the progression of renal injury in $\operatorname{Ig} \mathrm{AN}$ (41). In addition, a previous report showed that patients with IgAN exhibited increased serum levels of Th17 and Th17 family cytokines. In addition, serum levels of IL-17A and IL-21 were elevated in $\mathrm{IgAN}$, and serum IL-17A was correlated with 24-h proteinuria (42). Furthermore, a recent study reported that the number of tonsillar regulatory T-cells was decreased in patients with $\operatorname{Ig} \mathrm{AN}$, which was negatively correlated with the number of dimeric IgA-producing cells (43).

The JAK/STAT signaling pathway mediates the biological responses induced by numerous cytokines, and it is particularly important for differentiation of helper T-cells. Cytokines bind to the cell surface receptors of immune and non-immune cells to activate the JAK-STAT signaling pathway, which affects the function of $\mathrm{CD}^{-}{ }^{-} \mathrm{T}$-cells by upregulating the expression of specific target genes (44-46). In the present study, assays were performed to detect the levels of T-cell cytokines in serum samples and JAK/STAT pathway proteins in the PBMCs of all patients with $\operatorname{IgAN}$ prior to treatment and in the healthy control group (49 healthy subjects were selected as the normal control group) There was no significant difference in sex and 

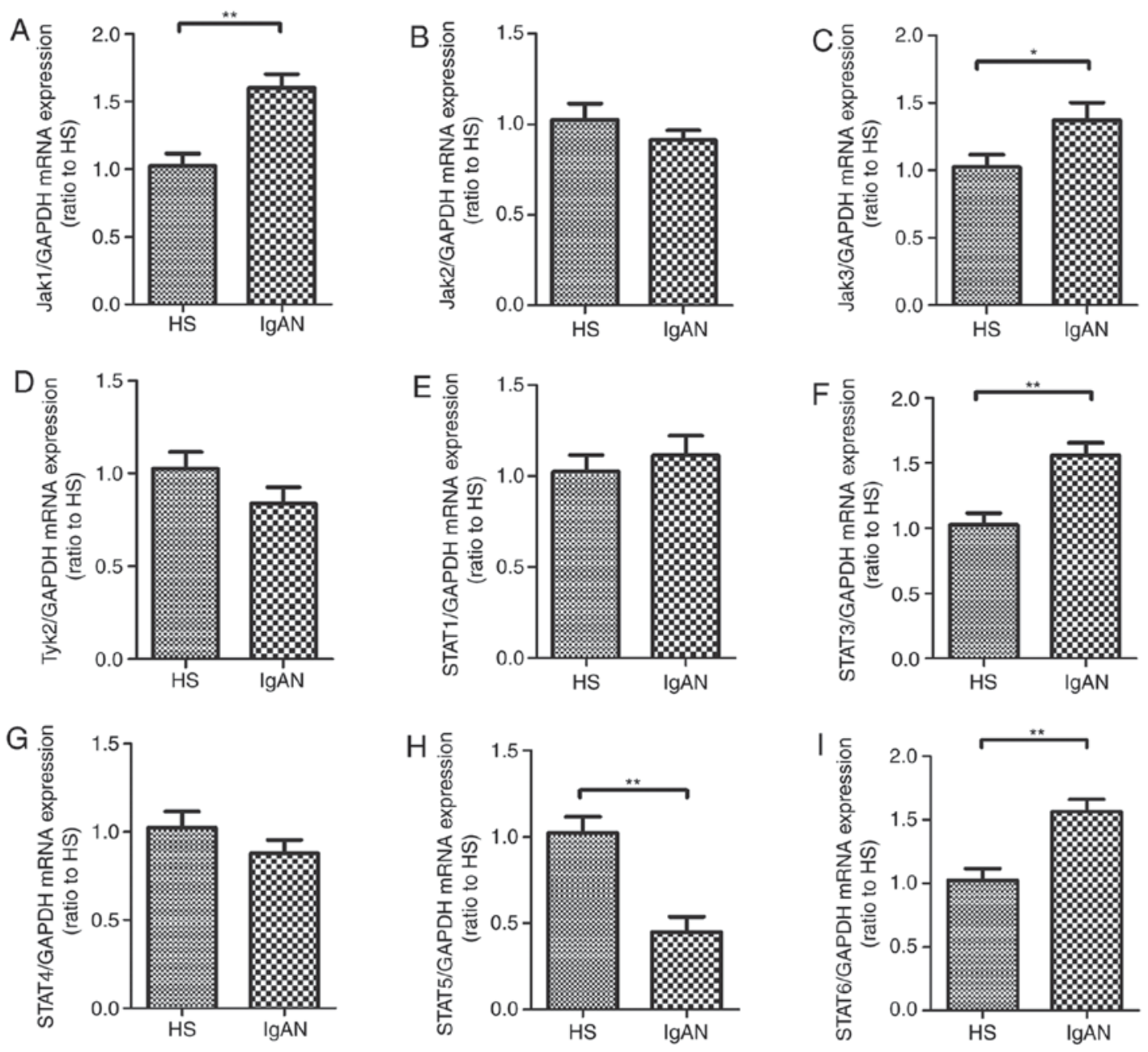

Figure 5. Expression of related molecules of JAK/STAT pathway in PBMCs from patients with IgAN. The expression of (A) Jak1, (B) Jak2, (C) Jak3, (D) Tyk2, (E) STAT1, (F) STAT3, (G) STAT4, (H) STAT5 and (I) STAT6 mRNA were measured in PBMCs from patients with IgAN and healthy controls. Data are presented as the mean \pm standard deviation $\left(" \mathrm{P}<0.05,{ }^{* *} \mathrm{P}<0.01\right)$. PBMCs. Peripheral blood mononuclear cells; JAK, Janus kinase; STAT, signal transducers and activators of transcription; HS, healthy subjects; IgAN, immunoglobulin A nephropathy; Tyk2, tyrosine kinase 2.

age between the patients with IgAN and the control group (Table I). The results revealed that higher levels of serum IL-4, IL-17, TGF- $\beta 1$ and IL-21 were detected in patients with IgAN compared with the normal controls. There was no significant difference in IFN- $\gamma$ expression between patients $\operatorname{Ig} A N$ and the normal control group. Furthermore, the patients with IgAN were treated for 1 month (AF-IgAN), and the serum levels of IL-4, IL-17, TGF- $\beta 1$ and IL-21 were decreased compared with the levels before treatment. Additionally, the results indicated that the expression of Jak1, Jak3, STAT3 and STAT6 mRNA was significantly upregulated in the PBMCs of patients with IgAN. However, STAT5 mRNA expression was decreased in the PBMCs of patients with IgAN. There was no significant difference in the expression of Jak2, Tyk2, STAT1 and STAT4 mRNA between patients with IgAN and the normal control group. These results indicated that the imbalance of T-cell-synthesized pro-inflammatory cytokines has a critical role in the development and progression of IgAN. Thus, T-lymphocytes have the potential to be involved in therapeutic intervention and a biomarker for the treatment and monitoring of this disease.

In summary, the results demonstrate that corticosteroid therapy is likely to be effective in patients IgAN with minimal proteinuria $(<1 \mathrm{~g} /$ day) and a high renal pathological score. The imbalance of dysregulation of $\mathrm{CD}^{+} \mathrm{T}$ cells subsets in IgAN may have a role in disease pathogenesis and progression, which is associated with the activation of the JAK/STAT signaling pathway. The levels of T-cell cytokines (IL-4, IL-17, TGF- $\beta 1$ and IL-21) may therefore represent novel therapeutic targets and biomarkers for the treatment and monitoring of IgAN.

\section{Acknowledgements}

Not applicable.

\section{Funding}

The present study was supported by Fudan University Affiliated Minhang Hospital level issues (no. 2017MHJC08) and the National Natural Science Foundation of China (no. 81774080).

\section{Availability of data and materials}

All data generated or analyzed during this study are included in this published article. 


\section{Authors' contributions}

YT, HH and XX conceived the study, designed the study protocol and wrote the paper. YT, $\mathrm{HH}$ and $\mathrm{PH}$ performed the experiments. WS and XC analyzed the data. YT and $\mathrm{HH}$ wrote the final version of the paper. All authors reviewed and approved the final version of the manuscript.

\section{Ethics approval and consent to participate}

All healthy and patient donors provided written informed consent prior to sampling. The experiments and procedures were conducted in accordance with the Helsinki Declaration of 1975 , and were approved by the Human Ethics Committee of School of Medicine, Fudan University.

\section{Patient consent for publication}

The patients in the present study agreed to publication of the anonymous data.

\section{Competing interests}

The authors declare that they have no competing interests.

\section{References}

1. Moresco RN, Speeckaert MM and Delanghe JR: Diagnosis and monitoring of IgA nephropathy: The role of biomarkers as an alternative to renal biopsy. Autoimmun Rev 14: 847-853, 2015.

2. Fabiano RC,Pinheiro SV and Simões E Silva AC: Immunoglobulin A nephropathy: A pathophysiology view. Inflamm Res 65: 757-770, 2016

3. KDIGO clinical practice guidelines for glomerulonephritis (2012) Chapter 10: Immunoglobulin A nephropathy. Kidney Int Suppl 2: S209-S217.

4. Glassock RJ: Glomerular disease: Targeted steroid therapy for IgA nephropathy. Nat Rev Nephron 13: 390-392, 2017.

5. Suzuki Y, Suzuki H, Makita Y, Takahata A, Takahashi K, Muto M, Sasaki Y, Kelimu A, Matsuzaki K, Yanagawa H, et al: Diagnosis and activity assessment of immunoglobulin A nephropathy: Current perspectives on noninvasive testing with aberrantly glycosylated immunoglobulin A-related biomarkers. Int J Nephrol Renovasc Dis 7: 409-414, 2014.

6. Yeo SC, Cheung CK and Barratt J: New insights into the pathogenesis of IgA nephropathy. Pediatr Nephrol 33: 763-777, 2018.

7. Robert T, Berthelot L, Cambier A, Rondeau E and Monteiro RC: Molecular insights into the pathogenesis of $\operatorname{IgA}$ nephropathy. Trends Mol Med 21: 762-775, 2015.

8. Hu S, Bao H, Xu X, Zhou X, Qin W, Zeng C and Liu Z: Increased miR-374b promotes cell proliferation and the production of aberrant glycosylated $\operatorname{Ig} \mathrm{A} 1$ in B cells of $\operatorname{Ig} \mathrm{A}$ nephropathy. FEBS Lett 589: 4019-4025, 2015.

9. Suzuki H, Allegri L, Suzuki Y, Hall S, Moldoveanu Z, Wyatt RJ, Novak J and Julian BA: Galactose-deficient IgA1 as a candidate urinary polypeptide marker of IgA nephropathy? Dis Markers 2016: 7806438, 2016.

10. Knoppova B, Reily C, Maillard N, Rizk DV, Moldoveanu Z, Mestecky J, Raska M, Renfrow MB, Julian BA and Novak J: The origin and activities of IgA1-containing immune complexes in IgA nephropathy. Front Immunol 7: 117, 2016.

11. Suzuki H, Fan R, Zhang Z, Brown R, Hall S, Julian BA, Chatham WW, Suzuki Y, Wyatt RJ, Moldoveanu Z, et al: Aberrantly glycosylated IgA1 in IgA nephropathy patients is recognized by $\operatorname{IgG}$ antibodies with restricted heterogeneity. J Clin Invest 119: 1668-1677, 2009.

12. Tomino Y, Sakai H, Miura M, Endoh M and Nomoto Y: Detection of polymeric IgA in glomeruli from patients with $\operatorname{Ig} \mathrm{A}$ nephropathy. Clin Exp Immunol 49: 419-425, 1982.
13. Lai KN, Ho RT, Leung JC, Lai FM and Li PK: Increased mRNA encoding for transforming factor-beta in CD4+ cells from patients with Ig Anephropathy. Kidney Int 46: 862-868, 1994.

14. Batra A, Smith AC, Feehally J and Barratt J: T-cell homing receptor expression in IgA nephropathy. Nephrol Nephrol Dial Transplant 22: 2540-2548, 2007.

15. Lai KN, Ho RT, Leung JC, Chui YL, Lim PL, Lui SF and Li PK: CD4-positive cells from patients with $\operatorname{IgA}$ nephropathy demonstrate increased mRNA of cytokines that induce the IgA switch and differentiation. J Pathol 174: 13-22, 1994.

16. Haas M: Histologic subclassification of IgA nephropathy: A clinicopathologic study of 244 cases. Am J Kidney Dis 6: 829-842, 1997.

17. Katafuchi R, Kiyoshi Y, Oh Y, Uesugi N, Ikeda K, Yanase T and Fujimi S: Glomerular score as a prognosticator in $\operatorname{IgA}$ nephropathy: Its usefulness and limitation. Clin Nephrol 49: 1-8, 1998.

18. Zhu Y, Ye X, Zhu B, Pei X, Wei L, Wu J and Zhao W: Comparisons between the 2012 New CKD-EPI (ChronicKidney Disease Epidemiology Collaboration) equations and other four approved equations. PLoS One 9: e84688, 2014.

19. Szeto CC and Li PK: MicroRNAs in IgA nephropathy. Nat Rev Nephrol 10: 249-256, 2014.

20. Rasche FM, Keller F, Rasche WG, Schiekofer S, Boldt A, Sack U and Fahnert J: Why, when and how should immunosuppressive therapy considered in patients with immunoglobulin A nephropathy? Clin Exp Immunol 186: 115-133, 2016.

21. Park KS, Han SH, Kie JH, Nam KH, Lee MJ, Lim BJ, Kwon YE, Kim YL, An SY, Kim CH, et al: Comparison of the Haas and the Oxford classifications for prediction of renal outcome in patients with IgA nephropathy. Hum Pathol 45: 236-243, 2014.

22. Lee SM, Rao VM, Franklin WA, Schiffer MS, Aronson AJ, Spargo BH and Katz AI: IgA nephropathy: Morphologic predictors of progressive renal disease. HUM Pathol 13: 314-22, 1982.

23. Haas M and Reich HN: Morphologic markers of progressive immunoglobulin A nephropathy. Adv Chronic Kidney Dis 19: 107-113, 2012.

24. Pozzi C: Treatment of IgA nephropathy. J Nephrol 29: 21-25, 2016.

25. Chakera A, MacEwen C, Bellur SS, Chompuk LO, Lunn D and Roberts ISD: Prognostic value of endocapillary hypercellularity in $\operatorname{IgA}$ nephropathy patients with no immunosuppression. J Nephrol 29: 367-375, 2016.

26. O'Shaughnessy MM and Lafayette RA: Corticosteroids for IgA Nephropathy: TESTING for Benefit, Discovering Harm. JAMA 318: 429-431, 2017.

27. Coppo R, Lofaro D, Camilla RR, Bellur S, Cattran D, Cook HT, Roberts IS, Peruzzi L, Amore A, Emma F, et al: Risk factors for progression in children and young adults with IgA nephropathy: An analysis of 261 cases from the VALIGA European cohort. Pediatr Nephrol 32: 139-150, 2017.

28. Eitner F, Ackermann D, Hilgers RD and Floege J: Supportive versus immunosuppressive therapy of progressive IgA nephropathy (STOP) IgAN trial: Rationale and study protocol. J Nephrol 21: 284-289, 2008

29. Nagy J, Sági B, Máté J, Vas T and Kovács T: Considerations on the treatment of IgA nephropathy on the basis of the results of the latest studies (STOP-IgAN, TESTING, NEFIGAN). Orv Hetil 158: 1946-1952, 2017 (In Hungarian).

30. Alvarado AS, Andeen NK, Brodsky S, Hinton A, Nadasdy T, Alpers CE, Blosser C, Najafian B and Rovin BH: Location of glomerular immune deposits, not codeposition of immunoglobulin $\mathrm{G}$, influences definitive renal outcomes in immunoglobulin A nephropathy. Nephrol Dial Transplant 33: 1168-1175, 2018.

31. Kiryluk K, Li Y, Moldoveanu Z, Suzuki H, Reily C, Hou P, Xie J, Mladkova N, Prakash S, Fischman C, et al: GWAS for serum galactose-deficient IgA1 implicates critical genes of the O-glycosylation pathway. PLoS Genet 13: e1006609, 2017.

32. Stuchlova Horynova M, Vrablikova A Stewart TJ, Takahashi K, Czernekova L, Yamada K, Suzuki H, Julian BA, Renfrow MB, Novak J and Raska M: N-acetylgalactosaminide $\alpha 2,6$-sialyltransferase II is a candidate enzyme for sialylation of galactose-deficient IgA1, the key autoantigen in IgA nephropathy. Nephrol Dial Transplant 30: 234-238, 2015.

33. Kiryluk K and Novak J: The genetics and immunobiology of IgA nephropathy. J Clin Invest 124: 2325-2332, 2014.

34. Ye M, Peng Y, Liu C, Yan W, Peng X, He L, Liu H and Liu F: Vibration induces BAFF overexpression and aberrant O-Glycosylation of IgA1 in cultured human tonsillar mononuclear cells in IgA nephropathy. Biomed Res Int 2016: 9125960, 2016. 
35. Ju T and Cummings RD: A unique molecular chaperone Cosmc required for activity of the mammalian core 1 beta 3-galactosyltransferase. Proc Natl Acad Sci USA 99: 16613-16618, 2002.

36. Meng H, Ohtake H, Ishida A, Ohta N, Kakehata S and Yamakawa M: IgA production and tonsillar focal infection in IgA nephropathy. J Clin Exp Hematop 52: 161-170, 2012.

37. Neurath MF, Finotto S and Glimcher LH: The role of Th1/Th2 polarization in mucosal immunity. Nat Med 8: 567-573, 2002.

38. He L, Peng Y,Liu H, Yin W, Chen X, Peng X, Shao J,Liu Y and Liu F: Activation of the interleukin-4/signal transducer and activator of transcription 6 signaling pathway and homeodomain-interacting protein kinase 2 production by tonsillar mononuclear cells in IgA nephropathy. Am J Nephrol 38: 321-332, 2013.

39. Liu L, Kou P, Zeng Q, Pei G, Li Y, Liang H, Xu G and Chen S: CD4+ T Lymphocytes, especially Th2 cells, contribute to the progress of renal fibrosis. Am J Nephrol 36: 386-396, 2012.

40. Yamada K, Kobayashi N, Ikeda T, Suzuki Y, Tsuge T, Horikoshi S, Emancipator SN and Tomino Y: Down-regulation of core 1 beta 1, 3-galactosylatransferase and Cosmc by Th2 cytokine alters O-glycosylation of IgA1. Nephrol Dial Transplant 25: 3890-3897, 2010 .
41. Suzuki H, Suzuki Y, Aizawa M, Yamanaka T, Kihara M, Pang H, Horikoshi S and Tomino Y: Th1 polarization in murine IgA nephropathy directed by bone marrow-derived cells. Kidney Int 72: 319-327, 2007.

42. Lin FJ, Jiang GR, Shan JP, Zhu C, Zou J and Wu XR: Imbalance of regulatory $\mathrm{T}$ cells to Th17 cells in Ig A nephropathy. Scand J Clin Lab Invest 72: 221-229, 2010.

43. Huang H, Peng Y, Liu H, Yang X and Liu F: Decreased CD4+CD25+ cells and increased dimeric IgA-producing cells in tonsils in IgA nephropathy. J Nephrol 23: 202-209, 2010.

44. Chen X, Tang Y, Zhang Y, Zhuo M, Tang Z, Yu Y and Zang G: Tapasin modification on the intracellular epitope HBcAg18-27 enhances HBV-specific CTL immune response and inhibits hepatitis B virus replication in vivo. Lab Invest 94: 478-490, 2014.

45. Quintás-Cardama A and Verstovsek S: Molecular pathways: Jak/STAT pathway: Mutations, inhibitors, and resistance. Clin Cancer Res 19: 1933-1940, 2013.

46. Shea-Donohue T, Fasano A, Smith A and Zhao A: Enteric pathogens and gut function: Role of cytokines and STATs. Gut Microbes 1: 316-324, 2010. 